\title{
Mixed estimates for singular integrals on weighted Hardy spaces
}

\author{
María Eugenia Cejas $^{1}$. Estefanía Dalmasso ${ }^{2}$
}

Received: 26 February 2019 / Accepted: 14 October 2019 / Published online: 4 November 2019

(c) Universidad Complutense de Madrid 2019

\begin{abstract}
In this paper we give quantitative bounds for the norms of different kinds of singular integral operators on weighted Hardy spaces $H_{w}^{p}$, where $0<p \leq 1$ and $w$ is a weight in the Muckenhoupt $A_{\infty}$ class. We deal with Fourier multiplier operators, CalderónZygmund operators of homogeneous type which are particular cases of the first ones, and, more generally, we study singular integrals of convolution type. In order to prove mixed estimates in the setting of weighted Hardy spaces, we need to introduce several characterizations of weighted Hardy spaces by means of square functions, LittlewoodPaley functions and the grand maximal function. We also establish explicit quantitative bounds depending on the weight $w$ when switching between the $H_{w}^{p}$-norms defined by the Littlewood-Paley-Stein square function and its discrete version, and also by applying the mixed bound $A_{q}-A_{\infty}$ result for the vector-valued extension of the HardyLittlewood maximal operator given in Buckley (Trans Am Math Soc 340(1):253-272, 1993).
\end{abstract}

Keywords Weighted Hardy spaces · Singular integrals · Mixed estimates · Calderón-Zygmund operators · Fourier multipliers

Mathematics Subject Classification 42B30 - 42B20 $\cdot$ 42B15 $\cdot 42 \mathrm{~B} 25$

Estefanía Dalmasso

edalmasso@santafe-conicet.gov.ar

María Eugenia Cejas

mec.eugenia@gmail.com

1 Departamento de Matemática, Facultad de Ciencias Exactas, Universidad Nacional de La Plata, CONICET, Calle 50 y 115, 1900 La Plata, Buenos Aires, Argentina

2 Instituto de Matemática Aplicada del Litoral, UNL, CONICET, FIQ., Predio Dr. Alberto Cassano del CCT-CONICET-Santa Fe, Colectora Ruta Nacional 168, S3007ABA Santa Fe, Argentina 


\section{Introduction}

Boundedness of singular integrals in weighted norms arises in the analysis of PDE's. It is a classical result that many operators in harmonic analysis are bounded on $L_{w}^{p}\left(\mathbb{R}^{n}\right)$, $1<p<\infty$, if the weight $w$ belongs to the so-called $A_{p}$ Muckenhoupt class. The sharp dependence of the corresponding $L_{w}^{p}\left(\mathbb{R}^{n}\right)$ operator norms in terms of the $A_{p}$ characteristic of $w$ has been studied for different operators in the last few years. We recall that a weight $w$, that is, a non-negative locally integrable function, belongs to the Muckenhoupt $A_{p}$ class for $1<p<\infty$ if

$$
[w]_{A_{p}}=\sup _{Q}\left(\frac{1}{|Q|} \int_{Q} w\right)\left(\frac{1}{|Q|} \int_{Q} w^{-\frac{1}{p-1}}\right)^{p-1}<\infty
$$

where the supremum is taken over all cubes in $\mathbb{R}^{n}$ with sides parallel to the axes. In the case $p=1$ we say that $w \in A_{1}$ if

$$
M w \leq k w \text { a.e. }
$$

and we define $[w]_{A_{1}}=\inf \{k>0: M w \leq k w$ a.e. $\}$. For $p=\infty$, we say that $w \in A_{\infty}$ if

$$
[w]_{A_{\infty}}=\sup _{Q} \frac{1}{w(Q)} \int_{Q} M\left(w \chi_{Q}\right)<\infty
$$

where $w(Q)=\int_{Q} w$. We call $[w]_{A_{p}}$ the $A_{p}$ constant or characteristic of the weight $w$. It is well-known that there exists a constant that depends on the dimension $n$ such that $[w]_{A_{\infty}} \leq c_{n}[w]_{A_{p}}$, for $p \geq 1$. All the above constants can be defined over balls instead of cubes, and they are the same up to a dimensional constant.

In [1], Buckley proved that for the Hardy-Littlewood maximal operator $M$ and $1<p<\infty$

$$
\|M\|_{L_{w}^{p}\left(\mathbb{R}^{n}\right)} \leq C[w]_{A_{p}}^{\frac{1}{p-1}}
$$

and this result is sharp in the sense that the right-hand side of (1.1) cannot be replaced by $\varphi\left([w]_{A_{p}}\right)$ for any positive nondecreasing function $\varphi$ growing more slowly than $t^{\frac{1}{p-1}}$. In the same article [1], it was shown that for the convolution Calderón-Zygmund singular integral operators $T$ and $1<p<\infty$,

$$
\|T\|_{L_{w}^{p}\left(\mathbb{R}^{n}\right)} \leq C[w]_{A_{p}}^{\frac{p}{p-1}}
$$

and the best power of $[w]_{A_{p}}$ is at least $\max \left\{1, \frac{1}{p-1}\right\}$. In the case $p=2$ and $T=H$ is the Hilbert transform, Petermichl and Pott improved it to the power $\frac{3}{2}$ (see [28]) and, later on, Petermichl in [25] obtained the best possible linear dependence for $p \geq 2$. For the case $1<p<2$, that dependence of the $[w]_{A_{p}}$ constant is a consequence of the sharp extrapolation proved in [5]. For the Riesz transforms, in [26], the author 
obtained the same linear bound. From [29], we also know that the linear dependence on the $A_{p}$ constant is the sharpest for the Ahlfors-Beurling operator.

Then, proving a linear dependence on the $A_{2}$ constant for general CalderónZygmund operators became an important problem in the area that was finally solved by Hytönen in [15]. This result was improved in [13] by means of what it is now called mixed $A_{p}-A_{\infty}$ estimates, as the ones obtained in that paper. Namely, for a Calderón-Zygmund operator $T$, Hytönen and Pérez showed in [13] that

$$
\|T\|_{L_{w}^{2}\left(\mathbb{R}^{n}\right)} \leq c[w]_{A_{2}}^{\frac{1}{2}} \max \left\{[w]_{A_{\infty}},\left[w^{-1}\right]_{A_{\infty}}\right\}^{\frac{1}{2}}
$$

Afterwards, this was extended for $p>1$ in [11].

When considering $p=1$, it is well-known that the above operators are unbounded in $L_{w}^{1}$, for $w \in A_{1}$, but a weak type estimate can be stated (see [21] and [13]) with the following mixed bound for the norm of the Calderón-Zygmund operator $T$

$$
\|T\|_{L_{w}^{1} \rightarrow L_{w}^{1, \infty}} \leq C[w]_{A_{1}}\left(1+\log [w]_{A_{\infty}}\right) .
$$

Given that the singular integrals we shall be dealing with are not bounded for $p=1$, a natural goal is to define a subspace of $L_{w}^{1}$ whose image under any singular integral is in $L_{w}^{1}$. With this purpose in mind, the Hardy space $H_{w}^{1}$ is introduced. Next, one can extend the definition for any $p>0$, obtaining the space $H_{w}^{p}$, where $w \in A_{q}$ for some $q>1$ (for more details, see the following sections). It is well-known that for $p>1$ these spaces coincide with $L_{w}^{p}$. Then, the case of interest is, actually, $0<p \leq 1$. Hardy spaces give rise to the so-called endpoints estimates for the $L_{w}^{p}$ boundedness of singular integrals, and this fact has motivated us to study the boundedness of singular integrals in $H_{w}^{p}$ for Muckenhoupt weights.

There are several ways to define Hardy spaces. According to each definition, different norms arise and, in most cases, they are equivalent up to constants that depend on the weight $w$. The weighted Hardy spaces were introduced by García-Cuerva in his Ph.D. dissertation [9]. A complete book about these spaces is [30] where the authors define the weighted Hardy spaces by using the non-tangential maximal functions. They introduce these spaces for doubling weights, namely, that there exists a constant $C>0$ such that $w(2 Q) \leq C w(Q)$ for any cube $Q$. Moreover, they study the boundedness of Calderón-Zygmund singular integrals on weighted Hardy spaces.

More recently, Lu and Zhu [22] apply a discrete version of Calderón's reproducing formula and Littlewood-Paley theory with weights to establish $H_{w}^{p}\left(\mathbb{R}^{n}\right) \rightarrow H_{w}^{p}\left(\mathbb{R}^{n}\right)$ $(0<p \leq \infty)$ boundedness results for singular integrals of convolution type when $w \in A_{\infty}$. Several authors have studied this problem requiring stronger hypotheses on the weight (see, for example, [18,20]).

The authors in [22, Theorem 1.1] show the boundedness of some sort of singular integrals in $H_{w}^{p}\left(\mathbb{R}^{n}\right)$ for $w \in A_{\infty}$. They claim that the bounds obtained in their article are quantitative but they fail in giving the explicit constant depending on the weight when switching from one $H_{w}^{p}$-norm to another (see [22, Proposition 2.1 and Corollary $2.1]$ ); there is actually a dependence on the $A_{\infty}$ constant of the weight $w$. Therefore, a difficulty in obtaining quantitative bounds for norms of operators in this setting is 
that one needs to be very careful when interchanging the norms in $H_{w}^{p}\left(\mathbb{R}^{n}\right)$ since there will be constants relating them that might depend on the weight. When one is just concerned about the boundedness of an operator, the constant can be disregarded.

In this article we shall precise the quantitative bounds obtained in [22]. While they provide bounds for the norm of the singular integral operator, they are not completely quantitative and accurate. In this sense, we first study multiplier operators $T_{m}$ where $m$ is a Fourier multiplier, and obtain bounds for $\left\|T_{m}\right\|_{H_{w}^{p} \rightarrow H_{w}^{p}}$ by means of the norm defined through a square function, where $w \in A_{\infty}$. In this case we obtain estimates for the same range of $p$ for which the boundedness of these operators in $H_{w}^{p}$ is well-known (see [30, Chapter XI, Theorem 6]). As a particular case of multiplier operators, we have the Calderón-Zygmund singular integrals of homogeneous type $T f=K * f$, where $K(x)=\frac{\Omega(x)}{|x|^{n}}$, provided that $\Omega$ is an homogeneous function of degree zero, infinitely differentiable on the unit sphere $S^{n-1}$ and $\int_{S^{n-1}} \Omega=0$. However, in Section 3 we are able to get quantitative estimates for those operators using the characterization of $H_{w}^{p}\left(\mathbb{R}^{n}\right)$ by the grand maximal function. This choice on the definition of the weighted Hardy space allows us to prove quantitative bounds for every $p \in(0,1]$. Using estimates for the grand maximal function given by Lerner and Wilson [19,33,34] we get mixed bounds for the norm in $H_{w}^{p}\left(\mathbb{R}^{n}\right)$ when $w \in A_{1}$. Next, as a consequence of an extrapolation result from [3] we can extend this last result for $w \in A_{\infty}$.

Afterwards, we consider singular integrals of convolution type with more general kernels that include the homogeneous type operators mentioned above. We follow the ideas by Lu and Zhu [22] and use the mixed bound $A_{q}-A_{\infty}$ for the vector-valued Hardy-Littlewood maximal operator obtained in [2], which is an improvement of the bound for this operator used by the authors. Moreover, we take special care of the equivalence between $H_{w}^{p}\left(\mathbb{R}^{n}\right)$ norms in order to get the correct dependence on the weight $w$.

\section{Quantitative bounds for multiplier operators with $A_{\infty}$ weights}

From now on, for simplicity, we will denote $H_{w}^{p}$ and $L_{w}^{p}$ instead of $H_{w}^{p}\left(\mathbb{R}^{n}\right)$ and $L_{w}^{p}\left(\mathbb{R}^{n}\right)$, respectively.

Set

$$
\Gamma_{\beta}(x)=\left\{(y, t) \in \mathbb{R}_{+}^{n+1}:|x-y|<t \beta\right\}, \quad \beta>0 .
$$

Let $P(x)=\frac{c_{n}}{\left(1+|x|^{2}\right)^{\frac{n+1}{2}}}$ be the Poisson kernel and, for $t>0$, set $P_{t}(x)=\frac{1}{t^{n}} P\left(\frac{x}{t}\right)$. Let $f \in \mathscr{S}$ and define $f(x, t)=P_{t} * f(x)$ its Poisson integral. If we consider the maximal function

$$
N_{\beta} f(x)=\sup _{(y, t) \in \Gamma_{\beta}(x)}|f(y, t)|
$$

the weighted Hardy space $H_{w}^{p}$ can be defined in terms of $N_{\beta}$ for $w \in A_{\infty}$ and any $\beta>0$. This means, $f \in H_{w}^{p}$ if and only if $N_{\beta} f \in L_{w}^{p}$. The norm is defined in the 
natural way $\|f\|_{H_{w}^{p}}:=\left\|N_{\beta} f\right\|_{L_{w}^{p}}$. We may refer to $H_{w}^{p}$ through the above definition, so the different equivalent norms used in our results will be related to it.

Let us denote by $\mathscr{S}$ the Schwartz class of functions and consider the subset $\hat{\mathscr{D}}_{0}:=$ $\{f \in \mathscr{S}: \operatorname{supp}(\hat{f})$ is compact and $0 \notin \operatorname{supp} \hat{f}\}$. A bounded function $m$ defined on $\mathbb{R}^{n} \backslash\{0\}$ is called a multiplier. A multiplier operator $T$ is defined by means of the Fourier transform as

$$
(T f)^{\widehat{N}}=m \hat{f}
$$

for $f \in \hat{\mathscr{D}}_{0}\left(\mathbb{R}^{n}\right)$, so $m$ is also called a Fourier multiplier. If $T$ is bounded on $H_{w}^{p}$, since $\hat{\mathscr{D}}_{0}$ is dense in $H_{w}^{p}$ for $w$ a doubling weight ([30, Chapter VII]), $T$ admits an extension as a continuous operator on $H_{w}^{p}$. We shall denote $T=T_{m}$ to indicate the corresponding Fourier multiplier of $T$.

In this section, we consider the following functions. We define the square function

$$
S_{\beta}(f)(x)=\left(\int_{\Gamma_{\beta}(x)}|t \nabla f(y, t)|^{2} d y \frac{d t}{t^{n+1}}\right)^{1 / 2},
$$

for $\beta>0$, and the Littlewood-Paley function

$$
g_{\mu}^{*}(f)(x)=\left(\int_{0}^{\infty} \int_{\mathbb{R}^{n}}\left(\frac{t}{t+|x-y|}\right)^{\mu n}|t \nabla f(y, t)|^{2} d y \frac{d t}{t^{n+1}}\right)^{\frac{1}{2}}
$$

for $\mu>1$, where $\nabla=\left(\partial_{x_{1}}, \ldots, \partial_{x_{n}}, \frac{d}{d t}\right)$.

Assume that $m \in C^{n+1}\left(\mathbb{R}^{n} \backslash\{0\}\right)$ and for any multi-index $\alpha=\left(\alpha_{1}, \ldots, \alpha_{n}\right) \in \mathbb{N}_{0}^{n}$ with $|\alpha|=\alpha_{1}+\cdots+\alpha_{n} \leq n+1$,

$$
\sup _{0<R<\infty} R^{2|\alpha|-n} \int_{R \leq|x| \leq 2 R}\left|D^{\alpha} m(x)\right|^{2} d x<\infty .
$$

This condition is known in the literature as $m \in M(2, n+1)$. Then, it is well-known (see [31, p. 233]) that

$$
S_{1}\left(T_{m} f\right)(x) \leq C g_{2+\frac{2}{n}}^{*}(f)(x), \quad x \in \mathbb{R}^{n},
$$

for any $f \in \hat{\mathscr{D}}_{0}$. This inequality will be the key ingredient in order to obtain quantitative bounds for $\left\|T_{m}\right\|_{H_{w}^{p} \rightarrow H_{w}^{p}}$.

As we said in the introduction, changing the $H_{w}^{p}$-norm for an equivalent one can lead to a different bound for the operator norm. Therefore, we shall fix a norm according to the operator we are dealing with. Since the $L_{w}^{p}$ norms of $N_{\beta}$ and $S_{\beta}$ are equivalent by [10, Theorem 1], we can also characterize $H_{w}^{p}$ using $S_{\beta}$, in particular, using $S_{1}$. Thus, in this section, we denote the weighted Hardy space by $\mathscr{H}_{w}^{p}$ and fix the norm $\|f\|_{\mathscr{H}_{w}^{p}}=\left\|S_{1}(f)\right\|_{L_{w}^{p}}, 0<p \leq 1$. Note that we choose to present this notation 
because it seemed to us that it made the exposition clearer. However, these space are all equivalent.

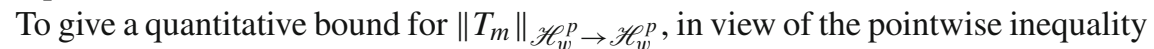
(2.1), it will be enough to prove that $g_{\mu}^{*}(f)$ can be estimated by $S_{1}(f)$ in norm $L_{w}^{p}$ for $0<p \leq 1$. This result is in the literature, more precisely, it can be found in [30, Theorem 4], but we shall revisit its proof in order to give the explicit constant depending on the weight. Our result is the following.

Theorem 1 Let $0<p \leq 1, w \in A_{\infty}$ and $0<r<\min \left\{\frac{p}{q_{w}}, 1\right\}$, where $q_{w}=\inf \{q>$ $\left.1: w \in A_{q}\right\}$. Then,

$$
\int_{\mathbb{R}^{n}}\left(g_{\mu}^{*}(f)(x)\right)^{p} w(x) d x \leq C(n, p, r, \mu)[w]_{A_{\frac{p}{r}}} \int_{\mathbb{R}^{n}}\left(S_{1}(f)(x)\right)^{p} w(x) d x
$$

for each $\mu>\frac{2}{r}$.

Therefore, we have the following quantitative bound for multiplier operators on $\mathscr{H}_{w}^{p}$, by combining (2.1) and theorem above. Notice that we can choose $r$ to verify $2+\frac{2}{n}>\frac{2}{r}$, that is, $r>\frac{n}{n+1}$. Thus, for $\frac{n}{n+1}<r<\min \left\{\frac{p}{q_{w}}, 1\right\}$, both inequalities hold. Moreover, if $r>\frac{1}{2}$, they are valid for any dimension.

Theorem 2 Let $\frac{n}{n+1} q_{w}<p \leq 1, w \in A_{\infty}$ and $0<r<\min \left\{\frac{p}{q_{w}}, 1\right\}$. Then, if $m \in M(2, n+1)$,

$$
\left\|T_{m}\right\|_{\mathscr{H}_{w}^{p} \rightarrow \mathscr{H}_{w}^{p}} \leq C[w]_{\frac{p}{r}}^{\frac{1}{p}},
$$

where the constant $C$ does not depend on the weight.

Proof of Theorem 1 Let us introduce some notation and properties that we will use to prove (2.2).

First, notice that, since $w \in A_{\infty}$, from the definition of $q_{w}$ there exists $r>\frac{n}{n+1}$ such that $w \in A_{\frac{p}{r}}$. Therefore, the weight satisfies the following doubling condition:

$$
w(B(x, a r)) \leq c_{d} a^{\frac{n p}{r}} w(B(x, r)), \quad \forall r>0, a \geq 1
$$

$c_{d}$ is called the doubling constant of $w$ and $B(x, r)$ denotes the ball centered at $x$ and radius $r$. It can be shown that

$$
c_{d}=\|M\|_{L_{w}^{\frac{p}{r}} \rightarrow L_{w}^{\frac{p}{r}, \infty}}^{\frac{p}{r}} \approx C(n, p, r)[w]_{A_{\frac{p}{r}}}
$$

which means that this weak type norm of the Hardy-Littlewood maximal operator is equivalent to the constant of the weight up to a constant that depends on $n, r$ and $p$ (see [1]). We shall also make use of the Hardy-Littlewood maximal operator associated to the measure $d v(x)=w(x) d x$ given by

$$
M_{w}(f)(x)=\sup _{B \ni x} \frac{1}{w(B)} \int_{B}|f(x)| w(x) d x, \quad f \in L_{\mathrm{loc}}^{1}(w),
$$


where the supremum is taken over the balls $B$ containing $x$. It is known that $M_{w}$ is of weak type $(1,1)$ respect to the measure $w(x) d x$ with constant $C_{n}$, only depending on $n$, not on $w$.

Let us proceed with the proof of (2.2). It is easy to see that

$$
g_{\mu}^{*}(f)(x) \leq C(\mu, n) \sum_{k=0}^{\infty} 2^{-k \mu n}\left(S_{2^{k}}(f)(x)\right)^{2}
$$

for every $\mu>1$ (c.f. [30, p. 54]).

Since $\frac{p}{2}<1$, we have

$$
\begin{aligned}
\int_{\mathbb{R}^{n}}\left(g_{\mu}^{*}(f)(x)\right)^{p} w(x) d x & \leq C(\mu, n) \int_{\mathbb{R}^{n}}\left(\sum_{k=0}^{\infty} 2^{-k \mu n}\left(S_{2^{k}}(f)(x)\right)^{2}\right)^{\frac{p}{2}} w(x) d x \\
& \leq C \int_{\mathbb{R}^{n}} \sum_{k=0}^{\infty} 2^{-k \mu n \frac{p}{2}}\left(S_{2^{k}}(f)(x)\right)^{p} w(x) d x \\
& =C \sum_{k=0}^{\infty} 2^{-\frac{k \mu n p}{2}} \int_{\mathbb{R}^{n}}\left(S_{2^{k}}(f)(x)\right)^{p} w(x) d x .
\end{aligned}
$$

We will show that the $L_{w}^{p}$-norm of $S_{2^{k}}$ can be replaced by the $L_{w}^{p}$-norm of $S_{1}$ times a constant that depends on the weight $w$.

Fix $k \geq 0$. Using a similar notation to [30], we define the level sets

$$
\mathscr{O}_{s}=\left\{x \in \mathbb{R}^{n}: S_{1}(f)(x)>s\right\}, \mathscr{O}_{s}^{\prime}=\left\{x \in \mathbb{R}^{n}: S_{2^{k}}(f)(x)>s\right\}
$$

and

$$
\mathscr{U}_{s}=\left\{x \in \mathbb{R}^{n}: M_{w}\left(\chi \mathscr{O}_{s}\right)(x)>\left(2 c_{d}\left(2^{k}\right)^{\frac{n p}{r}}\right)^{-1}\right\}
$$

Given $r, s>0$, we can write

$$
w\left(\mathscr{O}_{r s}^{\prime}\right) \leq w\left(\mathscr{U}_{s}\right)+w\left(\mathscr{O}_{r s}^{\prime} \cap \mathscr{U}_{s}^{c}\right)
$$

From the weak type $(1,1)$ of $M_{w}$,

$$
w\left(\mathscr{U}_{s}\right)=w\left(\left\{x \in \mathbb{R}^{n}: M_{w}\left(\chi_{\mathscr{O}_{s}}\right)(x)>\left(2 c_{d}\left(2^{k}\right)^{\frac{n p}{r}}\right)^{-1}\right\}\right) \leq C_{n} 2 c_{d} 2^{\frac{k n p}{r}} w\left(\mathscr{O}_{s}\right) .
$$

On the other hand,

$$
w\left(\mathscr{O}_{r s}^{\prime} \cap \mathscr{U}_{s}^{c}\right)=(r s)^{-2} \int_{\mathscr{O}_{r s}^{\prime} \cap \mathscr{U}_{s}^{c}}(r s)^{2} w(x) d x \leq(r s)^{-2} \int_{\mathscr{U}_{s}^{c}}\left(S_{2^{k}}(f)(x)\right)^{2} w(x) d x .
$$


From [30, Chapter IV, Lemma 7], we have that $\Gamma_{2^{k}}\left(\mathscr{U}_{s}^{c}\right) \subset \Gamma_{1}\left(\mathscr{O}_{s}^{c}\right)$, where $\Gamma_{\beta}(E):=$ $\cup_{x \in E} \Gamma_{\beta}(x)$, and also, from that result,

$$
w\left(B\left(y, 2^{k} t\right) \cap \mathscr{U}_{s}^{c}\right) \leq 2 c_{d} 2^{\frac{k n p}{r}} w\left(B(y, t) \cap \mathscr{O}_{s}^{c}\right), \quad t>0 .
$$

These two properties allow us to deduce the following bound for the integral on the right-hand side of (2.3):

$$
\begin{aligned}
& \int_{\mathscr{U}_{s}^{c}}\left(S_{2^{k}}(f)(x)\right)^{2} w(x) d x \\
& \quad=\int_{0}^{\infty} \int_{\Gamma_{2^{k}}\left(\mathscr{U}_{s}^{c}\right)}|t \nabla f(y, t)|^{2} w\left(B\left(y, 2^{k} t\right) \cap \mathscr{U}_{s}^{c}\right) d y \frac{d t}{t^{n+1}} \\
& \leq 2 c_{d} 2^{\frac{k n p}{r}} \int_{0}^{\infty} \int_{\Gamma_{1}\left(\mathscr{O}_{s}^{c}\right)}|t \nabla f(y, t)|^{2} w\left(B(y, t) \cap \mathscr{O}_{s}^{c}\right) d y \frac{d t}{t^{n+1}} \\
& =2 c_{d} 2^{\frac{k n p}{r}} \int_{\mathscr{O}_{s}^{c}}\left(S_{1}(f)(x)\right)^{2} w(x) d x .
\end{aligned}
$$

Thus, inserting this into (2.3),

$$
\begin{aligned}
w\left(\mathscr{O}_{r s}^{\prime} \cap \mathscr{U}_{s}^{c}\right) & \leq(r s)^{-2} 2 c_{d} 2^{\frac{k n p}{r}} \int_{\mathscr{O}_{s}^{c}}\left(S_{1}(f)(x)\right)^{2} w(x) d x \\
& =4 c_{d} 2^{\frac{k n p}{r}}(r s)^{-2} \int_{0}^{\infty} t w\left(\left\{t<S_{1}(f)(x) \leq s\right\}\right) d t \\
& \leq 4 c_{d} 2^{\frac{k n p}{r}}(r s)^{-2} \int_{0}^{s} t w\left(\mathscr{O}_{t}\right) d t
\end{aligned}
$$

Hence,

$$
w\left(\mathscr{O}_{r s}^{\prime}\right) \leq 4 c_{d} 2^{\frac{k n p}{r}}(r s)^{-2} \int_{0}^{s} t w\left(\mathscr{O}_{t}\right) d t+C_{n} 2 c_{d} 2^{\frac{k n p}{r}} w\left(\mathscr{O}_{s}\right)
$$

By [30, Chapter IV, Lemma 9] with $c_{1}=4 c_{d} 2^{\frac{k n p}{r}}$ and $c_{2}=C_{n} 2 c_{d} 2^{\frac{k n p}{r}}$,

$$
\int_{\mathbb{R}^{n}}\left(S_{2^{k}}(f)(x)\right)^{p} w(x) d x \leq C(n, p, r) c_{d} 2^{\frac{k n p}{r}} \int_{\mathbb{R}^{n}}\left(S_{1}(f)(x)\right)^{p} w(x) d x
$$

as we claimed.

Then,

$$
\begin{aligned}
\int_{\mathbb{R}^{n}}\left(g_{\mu}^{*}(f)(x)\right)^{p} w(x) d x & \leq C \sum_{k=0}^{\infty} 2^{-\frac{k \mu n p}{2}} \int_{\mathbb{R}^{n}}\left(S_{2^{k}}(f)(x)\right)^{p} w(x) d x \\
& \leq C(n, p, r) c_{d} \int_{\mathbb{R}^{n}}\left(S_{1}(f)(x)\right)^{p} w(x) d x \sum_{k=0}^{\infty} 2^{-\frac{k \mu n p}{2}} 2^{\frac{k n p}{r}}
\end{aligned}
$$




$$
\leq C(n, p, r)[w]_{A_{\frac{p}{r}}} \int_{\mathbb{R}^{n}}\left(S_{1}(f)(x)\right)^{p} w(x) d x
$$

where the series converges since $\mu>\frac{2}{r}$. The proof is now finished.

Remark 1 Note that in each step we are using the optimal constant $c_{d}$ for the doubling condition which give us a good constant in last theorem.

Remark 2 We also observe that the restriction on $p$, which translates in a restriction for $r$, coincides with the sufficient conditions given in [30, Chapter XI, Theorem 6] for the boundedness of $T_{m}$ on $\mathscr{H}_{w}^{p}$.

\section{Quantitative bounds for singular integrals of homogeneous type}

In this section, we first obtain quantitative bounds for the norm of $T$ on weighted Hardy spaces, when $w \in A_{1}$, for singular integrals of homogeneous type. Next, using an extrapolation argument, we will extend these bounds for $w \in A_{\infty}$.

We will need to introduce different functions in order to give the definition of the weighted Hardy spaces and several characterizations (see [35]).

For $f \in L_{\text {loc }}^{1}\left(\mathbb{R}^{n}\right)$ and $\alpha>0$, let

$$
A_{\alpha} f(y, t)=\sup _{\varphi \in \mathscr{C}_{\alpha}}\left|f * \varphi_{t}(y)\right|,
$$

where $\varphi_{t}(y)=t^{-n} \varphi\left(\frac{y}{t}\right)$ and $\mathscr{C}_{\alpha}$ is the collection of functions $\varphi: \mathbb{R}^{n} \rightarrow \mathbb{R}$ supported in the unit ball with integral zero, such that $\left|\varphi(y)-\varphi\left(y^{\prime}\right)\right| \leq\left|y-y^{\prime}\right|^{\alpha}$ for every $y, y^{\prime} \in \mathbb{R}^{n}$.

First, we define the square function of $f \in L_{\text {loc }}^{1}\left(\mathbb{R}^{n}\right)$ by

$$
S_{\psi, \beta}(f)(x)=\left(\int_{\Gamma_{\beta}(x)}\left|f * \psi_{t}(y)\right|^{2} \frac{d y d t}{t^{n+1}}\right)^{1 / 2}
$$

where $\psi \in C_{0}^{\infty}\left(\mathbb{R}^{n}\right)$ is radial, non trivial, supported in the unit ball with $\int \psi=0$ and such that

$$
\int_{0}^{\infty}|\hat{\psi}(\xi t)|^{2} \frac{d t}{t}=1, \quad \forall \xi \neq 0
$$

The intrinsic square function is defined as

$$
g_{\alpha, \mu}^{*}(f)(x)=\left(\int_{\mathbb{R}_{+}^{n+1}}\left(A_{\alpha}(f)(y, t)\right)^{2}\left(\frac{t}{t+|x-y|}\right)^{\mu n} \frac{d y d t}{t^{n+1}}\right)^{1 / 2}, \quad \alpha, \mu>0
$$


and the Littlewood-Paley function is given by

$$
g_{\phi, \mu}^{*}(f)(x)=\left(\int_{\mathbb{R}_{+}^{n+1}}\left|f * \phi_{t}(y)\right|^{2}\left(\frac{t}{t+|x-y|}\right)^{\mu n} \frac{d y d t}{t^{n+1}}\right)^{1 / 2}, \quad \mu>0
$$

where $\phi \in C_{0}^{\infty}\left(\mathbb{R}^{n}\right)$, is radial, with support contained in the ball $\{x:|x| \leq 1 / 2\}$ and $\int \phi=0$.

Finally, we define the grand maximal function $G_{N}$ for $f \in \mathscr{S}^{\prime}$, the set of tempered distributions. Given a natural number $N$, let $\mathscr{A}_{N}$ denote the set of all functions $\eta \in$ $C^{\infty}\left(\mathbb{R}^{n}\right)$ with supp $\eta \subset\{|x| \leq 1\}$ and

$$
\left\|\sum_{|\beta| \leq N}\left|D^{\beta} \eta\right|\right\|_{\infty} \leq 1
$$

where $\beta$ is a multi-index. Then

$$
G_{N}(f)(x):=\sup _{t>0} \sup _{\eta \in \mathscr{A}_{N}}\left|f * \eta_{t}(x)\right|
$$

It is well-known that one can define the weighted Hardy space for $0<p<\infty$, as the set

$$
\mathscr{H}_{w}^{p}=\left\{f \in \mathscr{S}^{\prime}: G_{N}(f) \in L_{w}^{p}\right\}
$$

The norm is given by $\|f\|_{\mathscr{H}_{w}^{p}}:=\left\|G_{N}(f)\right\|_{L_{w}^{p}}$. Note that this definition of $H_{w}^{p}$ is possible according to $[24,36]$. We fix the norm given by $G_{N}$ for the results of the present section.

We will deal with homogeneous type operators. Let us consider $\Omega$ an homogeneous function of degree 0 , infinitely differentiable on the unit sphere with

$$
\int_{|x|=1} \Omega(x) d \sigma(x)=0 .
$$

We consider the following singular integral operator given by

$$
T f(x):=\lim _{\varepsilon \rightarrow 0} \int_{|y|>\varepsilon} \frac{\Omega(y)}{|y|^{n}} f(x-y) d y .
$$

These type of operators are known as homogeneous singular integrals and they are particular cases of multiplier operators. More precisely, if $\Omega$ is as above, there exists a multiplier (a bounded function outside the origin) $m \in C^{\infty}\left(S^{n-1}\right)$ which is homogeneous of degree 0 (see [31, Chapter III, Theorem 6]). Thus, under some extra assumptions on $\Omega$, one can have $m \in M(2, n+1)$ (see [17] or [30]) and $T$ will be in the conditions of the previous section. We will show now that with no other hypotheses on $\Omega$ than the given above, we get mixed estimates and reach all the values of $p \in(0,1]$. 
We state the following pointwise estimate that holds for singular integrals and it can be found in [33] (see also [19]). It relates the square function of the homogeneous singular integral applied to $f$ with the Littlewood-Paley function of $f$.

Theorem 3 [33] Let us consider an homogeneous singular integral operator $T$ as defined above.

Let $\phi \in C^{\infty}\left(\mathbb{R}^{n}\right)$ be radial, $\operatorname{supp}(\phi) \subset\{|x| \leq 1 / 2\}$, satisfying that there exist positive constants $c$ and $\xi$ such that

$$
\int_{s}^{\infty}|\hat{\phi}(t, 0, \ldots, 0)|^{2} \frac{d t}{t} \geq c(1+s)^{-\xi}
$$

for every $s>0$, and that $\int P(x) \phi(x) d x=0$ for every polynomial $P$ of degree $\leq 2 n$. Let $\psi=\phi * \phi$. There exists a constant $C(\beta, \mu, \phi)$ and $\mu>3$ such that for all $\beta>0$

$$
S_{\psi, \beta}(T f) \leq C(\beta, \mu, \phi) g_{\phi, \mu}^{*}(f) .
$$

We will use the following result given by Lerner [19] in order to obtain bounds for the norms on weighted Hardy spaces.

Proposition 1 [19] Let $\psi$ be as in the definition of the square function and also verifying (3.2). Then there exist $N \in \mathbb{N}$ depending on $\xi$ and $n$, and $\beta$ depending on $n$ such that for any weight $w$ and for any $f$ with $G_{N}(f) \in L_{w}^{p}$,

$$
\left\|G_{N}(f)\right\|_{L_{w}^{p}} \leq c[w]_{A_{\infty}}^{\frac{1}{2}}\left\|S_{\psi, \beta}(f)\right\|_{L_{w}^{p}}
$$

where $c$ does not depend on either $f$ or $w$.

Remark 3 This result was obtained by Wilson in [33] but in [19] the author showed the bound in terms of $[w]_{A_{\infty}}$ which was defined in the Introduction. Inequality (3.4) with $f$ instead of $G_{N}(f)$ is essentially contained in [33].

We will obtain a mixed $A_{1}-A_{\infty}$ estimate for the norm $\|T\|_{\mathscr{H}_{w}^{p} \rightarrow \mathscr{H}_{w}^{p}}$ when $0<$ $p \leq 1$. For that, we shall use the following weighted inequality.

Lemma 1 [34] Let $0<p \leq 1$. There exists $\tilde{\mu}=\tilde{\mu}(p)>0$ such that for any $\mu>\tilde{\mu}$ and any weight $w$ we have

$$
\left(\int_{\mathbb{R}^{n}} g_{\phi, \mu}^{*}(f)^{p} w(x) d x\right)^{\frac{1}{p}} \leq C(n, N, p)\left(\int_{\mathbb{R}^{n}}\left|G_{N}(f)\right|^{p} M w(x) d x\right)^{\frac{1}{p}}
$$

where the constant does not depend on $w$.

Remark 4 If we consider $w \in A_{1}$, by the lemma above we obtain

$$
\left(\int_{\mathbb{R}^{n}} g_{\phi, \mu}^{*}(f)^{p} w(x) d x\right)^{\frac{1}{p}} \leq C(n, N, p)[w]_{A_{1}}^{\frac{1}{p}}\left(\int_{\mathbb{R}^{n}}\left|G_{N}(f)\right|^{p} w(x) d x\right)^{\frac{1}{p}}
$$

where the constant does not depend on $w$. 
Theorem 4 If $0<p \leq 1, w \in A_{1}$ and $T$ is a singular integral as in Theorem 3, then

$$
\|T\|_{\mathscr{H}_{w}^{p} \rightarrow \mathscr{H}_{w}^{p}} \leq C[w]_{A_{1}}^{\frac{1}{p}}[w]_{A_{\infty}}^{\frac{1}{2}}
$$

where the constant $C$ does not depend on the weight.

Proof Let $\phi$ and $\psi$ be as in Theorem 3. Then, for this choice of $\psi$, from Proposition 1 there exists $N \in \mathbb{N}$ such that

$$
\left\|G_{N}(T f)\right\|_{L_{w}^{p}} \leq C[w]_{A_{\infty}}^{\frac{1}{2}}\left\|S_{\psi, \beta}(T f)\right\|_{L_{w}^{p}}
$$

where the constant $C$ does not depend on $w$. Now, using the pointwise inequality (3.3) and last remark, with $\mu>\max \{3, \tilde{\mu}\}$, for $\tilde{\mu}$ as in Lemma 1 , we have

$$
\left\|S_{\psi, \beta}(T f)\right\|_{L_{w}^{p}} \leq C(\beta, \mu, \phi)\left\|g_{\phi, \mu}^{*}(f)\right\|_{L_{w}^{p}} \leq C(\beta, \mu, \phi, n, N, r)[w]_{A_{1}}^{\frac{1}{p}}\left\|G_{N}(f)\right\|_{L_{w}^{p}},
$$

so we obtain the desired inequality.

Now we will extend inequality (3.6) to the case of $A_{\infty}$ weights.

Proposition 2 If $0<p \leq 1, w \in A_{\infty}$. Let $0<r<\min \left\{\frac{p}{q_{w}}, 1\right\}$. Then, there exists $\tilde{\mu}=\tilde{\mu}(p)>0$ such that for any $\mu>\tilde{\mu}$, we have

$$
\left(\int_{\mathbb{R}^{n}} g_{\phi, \mu}^{*}(f)^{p} w(x) d x\right)^{\frac{1}{p}} \leq C(n, N, p, r)[w]_{A_{\frac{p}{r}}^{\frac{1}{p}}}^{\frac{1}{r}}[w]_{A_{\infty}}^{\frac{1}{r}-\frac{1}{p}}\left(\int_{\mathbb{R}^{n}}\left|G_{N}(f)\right|^{p} w(x) d x\right)^{\frac{1}{p}} .
$$

Proof We will give the proof in order to obtain the explicit constant in the inequality (3.7). However, the result is an immediate consequence of [3, Theorem 2.2].

We first notice that, since $w \in A_{\infty}$, from the definition of $r$ we have that $w \in A_{\frac{p}{r}}$. We denote $q=\frac{p}{r}$.

We are going to use Rubio de Francia's algorithm (see [9]). Define the operator $\mathscr{R}$, for $f \in L_{\text {loc }}^{1}\left(\mathbb{R}^{n}\right)$, by

$$
\mathscr{R} h(x)=\sum_{k=0}^{\infty} \frac{M^{k} h(x)}{2^{k}\|M\|_{L_{w^{1-q^{\prime}}}^{q^{\prime}}}^{k}}
$$

where $M^{k}$ is the Hardy-Littlewood maximal operator iterated $k$ times if $k \geq 1$ and, for $k=0$ is just the identity operator. It is immediate from the definition of $\mathscr{R}$ that

(a) $h(x) \leq \mathscr{R} h(x)$,

(b) $\|\mathscr{R} h\|_{L_{w^{1-q^{\prime}}}^{q^{\prime}}} \leq 2\|h\|_{L_{w^{1-q^{\prime}}}^{q^{\prime}}}$,

(c) $M(\mathscr{R} h)(x) \leq 2\|M\|_{L_{w^{1-q^{\prime}}}^{q^{\prime}}} \mathscr{R} h(x)$, meaning that $\mathscr{R} h \in A_{1}$ with constant independent of $h$. 
We write

$$
\left\|g_{\phi, \mu}^{*}(f)\right\|_{L_{w}^{p}}^{r}=\left\|\left(g_{\phi, \mu}^{*} f\right)^{r}\right\|_{L_{w}^{q}}=\sup _{h} \int_{\mathbb{R}^{n}}\left(g_{\phi, \mu}^{*} f(x)\right)^{r} h(x) w(x) d x,
$$

where the supremum is taken over all non-negative functions $h \in L_{w}^{q^{\prime}}$ with $\|h\|_{L_{w}^{q^{\prime}}}=1$.

For any of such functions $h$, we have that $h w \in L_{w^{1-q^{\prime}}}^{q^{\prime}}$ with $\|h w\|_{L_{w^{1-q^{\prime}}}^{q^{\prime}}}=\|h\|_{L_{w}^{q^{\prime}}}=$ 1.

Let us fix $h \in L_{w}^{q^{\prime}}$ with $\|h\|_{L_{w}^{q^{\prime}}}=1$. Then, from (a) and (c) with $h w$ and (3.6), we have

$$
\begin{aligned}
\int_{\mathbb{R}^{n}}\left(g_{\phi, \mu}^{*} f(x)\right)^{r} h(x) w(x) d x & \leq \int_{\mathbb{R}^{n}}\left(g_{\phi, \mu}^{*} f(x)\right)^{r} \mathscr{R}(h w)(x) d x \\
& \leq C(n, N, r)[\mathscr{R}(h w)]_{A_{1}} \int_{\mathbb{R}^{n}}\left|G_{N} f(x)\right|^{r} \mathscr{R}(h w)(x) d x .
\end{aligned}
$$

Note that, if $F \in L_{w}^{p}$ and $\mathscr{R}(h w) \in L_{w^{1-q^{\prime}}}^{q^{\prime}}, 0<w<\infty$ almost everywhere in the set where $f^{r} \mathscr{R}(h w)>0$. Then, by applying Hölder's inequality with $q$ and $q^{\prime}$, we get

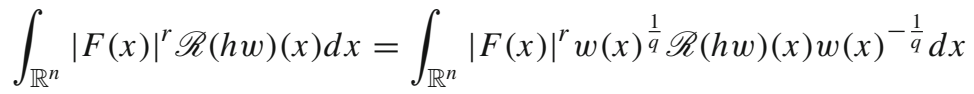

$$
\begin{aligned}
& \leq\|F\|_{L_{w}^{p}}^{r}\|\mathscr{R}(h w)\|_{L_{w^{1-q^{\prime}}}^{q^{\prime}}} \\
& \leq\|F\|_{L_{w}^{p}}^{r} 2\|h w\|_{L_{w^{1-q^{\prime}}}^{q^{\prime}}}=2\|F\|_{L_{w}^{p}}^{r}
\end{aligned}
$$

where we have used (b) and that $\|h w\|_{L_{w^{1-q^{\prime}}}^{q^{\prime}}}=1$.

Particularly, we can apply the inequality above to $F=G_{N}(f)$, which we assume in $L_{w}^{p}$ since, otherwise, there is nothing to prove. Thus, we get

$$
\int_{\mathbb{R}^{n}}\left(g_{\phi, \mu}^{*} f(x)\right)^{r} h(x) w(x) d x \leq C[\mathscr{R}(h w)]_{A_{1}}\left(\int_{\mathbb{R}^{n}}\left|G_{N} f(x)\right|^{p} w(x) d x\right)^{\frac{r}{p}} .
$$

In other words, we have proved that

$$
\left(\int_{\mathbb{R}^{n}} g_{\phi, \mu}^{*}(f)^{p} w(x) d x\right)^{\frac{1}{p}} \leq C[\mathscr{R}(h w)]_{A_{1}}^{\frac{1}{r}}\left(\int_{\mathbb{R}^{n}}\left|G_{N}(f)\right|^{p} w(x) d x\right)^{\frac{1}{p}} .
$$

Now, we need to estimate $[\mathscr{R}(h w)]_{A_{1}}$ in terms of the weight $w$. According to (c), we have that $[\mathscr{R}(h w)]_{A_{1}} \leq 2\|M\|_{L_{w^{1-q^{\prime}}}^{q^{\prime}}}$. But, the $A_{q^{\prime}}-A_{\infty}$ mixed estimate for the Hardy-Littlewood maximal operatoris known, see [13, Corollary 1.10]. Hence, 
$[\mathscr{R}(h w)]_{A_{1}} \leq c_{n} q\left(\left[w^{1-q^{\prime}}\right]_{A_{q^{\prime}}}[w]_{A_{\infty}}\right)^{\frac{1}{q^{\prime}}}=c_{n} \frac{p}{r}[w]_{A_{q}}^{\frac{1}{q}}[w]_{A_{\infty}}^{\frac{1}{q^{\prime}}}=c_{n} \frac{p}{r}[w]_{A_{\frac{p}{r}}^{\frac{r}{p}}}[w]_{A_{\infty}}^{1-\frac{r}{p}}$,

where we have used that $[w]_{A_{q}}=\left[w^{1-q^{\prime}}\right]_{A_{q^{\prime}}}^{q-1}$. Therefore,

$$
[\mathscr{R}(h w)]_{A_{1}}^{\frac{1}{r}} \leq c_{n}^{\frac{1}{r}}\left(\frac{p}{r}\right)^{\frac{1}{r}}[w]_{A_{\frac{p}{r}}^{\frac{1}{p}}}^{\frac{1}{p}}[w]_{A_{\infty}}^{\frac{1}{r}-\frac{1}{p}}
$$

which completes the proof.

Remark 5 An interesting open problem is to find the optimal value of $q$ such that $w \in A_{q}$ in terms of the constant $[w]_{A_{\infty}}$. It was proved in [14] that $q>e^{c_{n}[w]_{A_{\infty}}}$, with $[w]_{A_{q}} \leq e^{e^{c_{n}[w]} A_{\infty}}$, where $c_{n}$ is a dimensional constant. These type of embedding results has been studied in $[7,16,23,27,32]$, and, for the one-dimesional case, they are sharper.

By reproducing the proof of Theorem 4 with (3.7) instead of (3.6), we obtain the following quantitative mixed bound for $\|T\|_{\mathscr{H}_{w}^{p} \rightarrow \mathscr{H}_{w}^{p}}$.

Theorem 5 If $0<p \leq 1, w \in A_{\infty}$ and $T$ is a singular integral of homogeneous type, then

$$
\|T\|_{\mathscr{H}_{w}^{p} \rightarrow \mathscr{H}_{w}^{p}} \leq C[w]_{A_{\frac{p}{r}}^{\frac{1}{p}}}[w]_{A_{\infty}}^{\frac{1}{2}+\frac{1}{r}-\frac{1}{p}}
$$

where the constant $C$ does not depend on the weight.

Remark 6 Note that in last theorem the quantitative constant is bigger than the one proved in Theorem 2. However, for the operators of homogeneous type considered in this section the bounds can be obtained for every $p \in(0,1]$.

\section{Quantitative bounds for singular integrals of convolution type}

In this section we obtain a quantitative bound for the $H_{w}^{p} \rightarrow H_{w}^{p}$ boundedness of singular integrals of convolution type. The idea is to use a Calderón reproducing formula and to improve the bound for the vector valued maximal Hardy-Littlewood operator given in $[22,(2.2)]$. In fact we use an $A_{q}-A_{\infty}$ mixed estimate obtained in [2] which is a sharp bound.

Let $\psi$ be a Schwartz function on $\mathbb{R}^{n}$ which satisfies

$$
\int_{\mathbb{R}^{n}} \psi(x) x^{\alpha} d x=0
$$

for all multi-indexes $\alpha$ and

$$
\sum_{j \in \mathbb{Z}}\left|\hat{\psi}\left(2^{-j} \xi\right)\right|^{2}=1
$$


for all $\xi \neq 0$.

Definition 1 A function $f$ defined on $\mathbb{R}^{n}$ is said to be in $\mathscr{S}_{M}\left(\mathbb{R}^{n}\right)$ where $M$ is a positive integer, if there exists $C>0$ such that $f$ satisfies the following conditions:

(1) For $|\alpha| \leq M-1$,

$$
\left|D^{\alpha} f(x)\right| \leq C \frac{1}{(1+|x|)^{n+M+|\alpha|}}
$$

(2) For $\left|x-x^{\prime}\right| \leq \frac{1}{2}(1+|x|)$ and $|\gamma|=M$

$$
\left|D^{\gamma} f(x)-D^{\gamma} f\left(x^{\prime}\right)\right| \leq C \frac{\left|x-x^{\prime}\right|}{(1+|x|)^{n+2 M}}
$$

(3) For $|\alpha| \leq M-1$

$$
\int_{\mathbb{R}^{n}} f(x) x^{\alpha} d x=0 .
$$

If $f \in \mathscr{S}_{M}\left(\mathbb{R}^{n}\right)$ its norm is then defined by

$$
\|f\|_{\mathscr{S}_{M}\left(\mathbb{R}^{n}\right)}:=\inf \{C:(1) \text { and (2) hold }\}
$$

To simplify notation we denote $\mathscr{S}_{M}\left(\mathbb{R}^{n}\right)$ by $\mathscr{S}_{M}$. For $f \in\left(\mathscr{S}_{M}\right)^{\prime}$, the dual space of $\mathscr{S}_{M}$, define the Littlewood-Paley-Stein square function of $f$ by

$$
g(f)(x)=\left(\sum_{j \in \mathbb{Z}}\left|\psi_{j} * f(x)\right|^{2}\right)^{\frac{1}{2}}
$$

where $\psi_{j}(x)=2^{-j n} \psi\left(2^{-j} x\right)$, with $\psi$ as above.

For convenience, in this section we will modify the definition of the weighted Hardy space introduced in (3.1).

Definition 2 Let $0<p<\infty, w \in A_{\infty}$. Let $M=\left[\left(\frac{2 q_{w}}{p}-1\right) n\right]+1$, where $[\cdot]$ denotes the integer function. We define the weighted Hardy spaces as follows

$$
\mathbb{H}_{w}^{p}=\left\{f \in\left(\mathscr{S}_{M}\right)^{\prime}: g(f) \in L_{w}^{p}\right\}
$$

and the norm of $f$ in $\mathbb{H}_{w}^{p}$ is defined by

$$
\|f\|_{\mathbb{H}_{w}^{p}}=\|g(f)\|_{L_{w}^{p}\left(\mathbb{R}^{n}\right)} .
$$

Remark 7 In [6], the authors showed that the spaces $H_{w}^{p}$ and $\mathbb{H}_{w}^{p}$, coincide (see [6, Theorem 2.1 and Remark 2.1]). Moreover, they proved (see [6, Proposition 3.2]) that the space $\mathscr{S}_{M}$ is dense in $\mathbb{H}_{w}^{p}$ for any $A_{\infty}$ weight and $0<p<\infty$. 
Let $\phi \in C_{0}^{\infty}\left(\mathbb{R}^{n}\right)$ be supported in the unit ball and satisfying the same conditions as $\psi$ for a fixed $M_{0} \geq M$, where $M$ is the same as in the definition of $\mathbb{H}_{w}^{p}$.

For $j \in \mathbb{Z}$, we will consider the set $\mathscr{D}_{j}$ of dyadic cubes $Q$ of $\mathbb{R}^{n}$ with sides parallel to the coordinate axes and side-length $\ell(Q)=2^{-j}$.

We define the discrete Littlewood-Paley function

$$
g_{d}(f)(x)=\left(\sum_{j \in \mathbb{Z}} \sum_{Q \in \mathscr{D}_{j+N}}\left|\phi_{j} * f\left(x_{Q}\right)\right|^{2} \chi_{Q}(x)\right)^{\frac{1}{2}}
$$

where $x_{Q}$ is a point in $Q, \phi_{j}(x)=2^{-j n} \phi\left(2^{j} x\right)$ and $N$ is some fixed large integer. We reserve the notation $\phi$ for $g_{d}$.

We will consider singular integrals of convolution type $T f=K * f$ so that the kernel $K$ satisfies

(1) For all multi-indexes $\alpha$, and $x \neq 0$

$$
\left|D^{\alpha} K(x)\right| \leq C_{\alpha}|x|^{-n-|\alpha|}
$$

(2) For any $0<\varepsilon<N<\infty$

$$
\left|\int_{\varepsilon<|x|<N} K(x) d x\right| \leq C .
$$

The next two lemmas involving functions on $\mathscr{S}_{M}$ were proved in [22], and will be useful in the proof of Theorem 7.

Lemma 2 [22] If $\psi, \varphi \in \mathscr{S}_{M}$, for any given positive integers $a$ and $b$, there exists $a$ constant $C$ depending only on $a$ and $b$, such that

$$
\left|\psi_{i} * \varphi_{j}(x)\right| \leq C\left(\frac{i}{j} \wedge \frac{j}{i}\right)^{a} \frac{(i \vee j)^{b}}{(i \vee j+|x|)^{n+b}}, \quad \forall x \in \mathbb{R}^{n}
$$

Lemma 3 [22] Let $\psi \in \mathscr{S}_{M}, i, j \in \mathbb{Z}$ and let $Q$ be a dyadic cube in $\mathscr{D}_{j+N}$. Set $a, b \in \mathbb{N}$. Then, for any $u, u^{*} \in Q$ and any $r$ satisfying $\frac{n}{n+b}<r \leq 1$, we have

$$
\begin{aligned}
& \sum_{W \in \mathscr{D}_{i+N}} \frac{2^{-|j-i| a}|W| 2^{-(j \wedge i) b}}{\left(2^{-j \wedge i}+\left|u-x_{W}\right|\right)^{n+b}}\left|\psi_{i} * f\left(x_{W}\right)\right| \\
& \quad \leq C 2^{-|j-i| a} 2^{\left(\frac{1}{r}-1\right) n\left(N+(i-j)_{+}\right)} M_{r}\left(\sum_{W \in \mathscr{D}_{i+N}}\left|\psi_{i} * f\left(x_{W}\right)\right| \chi_{W}\right)\left(u^{*}\right),
\end{aligned}
$$

where $(i-j)_{+}=\max \{i-j, 0\}, x_{W}$ is a fixed point of the cube $W, M_{r} h=M\left(|h|^{r}\right)^{1 / r}$ and $C$ is a constant depending on the dimension $n$. 
We will need the following discrete Calderón's reproducing formula, proved in [22, Theorem 2.1] (c.f. [8] or [12]).

Theorem 6 [22] Suppose that $\psi_{j}$ is as in the definition of $g$. Then for any $M \geq 1$, we can choose a large $N$ depending on $M$ and $\psi$ such that the following discrete Calderón's reproducing identity

$$
f(x)=\sum_{j \in \mathbb{Z}} \sum_{Q \in \mathscr{D}_{j+N}}|Q| \tilde{\psi}_{j}\left(x-x_{Q}\right) \psi_{j} * f\left(x_{Q}\right)
$$

holds in $\mathscr{S}_{M}$ and in the dual space $\left(\mathscr{S}_{M}\right)^{\prime}$, where $\widetilde{\psi}_{j}\left(\cdot-x_{Q}\right) \in \mathscr{S}_{M}$ for each $Q$ and $x_{Q}$ is any fixed point in $Q$.

Next, we give the aforementioned sharp weighted estimate for the vector-valued extension of the Hardy-Littlewood maximal operator given in [2].

Lemma 4 [2] Let $1<p, q<\infty$ and let $w$ be a weight in $A_{p}$. We define the vectorvalued Hardy-Littlewood maximal operator $\bar{M}_{q}$ as

$$
\bar{M}_{q} f(x)=\left(\sum_{j=1}^{\infty} M f_{j}(x)^{q}\right)^{\frac{1}{q}} .
$$

Then,

$$
\left\|\overline{M_{q}} f\right\|_{L_{w}^{p}} \leq C[w]_{A_{p}}^{\frac{1}{p}}\left([w]_{A_{\infty}}^{\left(\frac{1}{q}-\frac{1}{p}\right)_{+}}+[\sigma]_{A_{\infty}}^{\frac{1}{p}}\right)\left\||f|_{q}\right\|_{L_{w}^{p}}
$$

where

$$
|f|_{q}(x)=\left(\sum_{j=1}^{\infty}\left|f_{j}(x)\right|^{q}\right)^{\frac{1}{q}}
$$

and we denote $\sigma=w^{-\frac{1}{p-1}}$ the dual weight of $w$ in $A_{p}$.

This result improved Theorem 1.12 from [4] since, as we stated in the introduction, $[w]_{A_{\infty}} \leq c_{n}[w]_{A_{q}}$, for $c_{n}$ a dimensional constant.

We will use the following inequality between the norms of Littlewood-Paley square functions.

Note that in [22], the authors mention that the norms of $g_{d}$ and $g$ in $L_{w}^{p}$ are equivalent, but this is true up to a constant that depends on the weight. For our purposes, the equivalence is not needed, so we just write here the inequality that it is useful for us with the explicit dependence on the weight.

Lemma 5 Let $w \in A_{\infty}$ and $\frac{n}{n+M}<r<\min \left\{\frac{p}{q_{w}}, 1\right\}$, then

$$
\left\|g_{d}(f)\right\|_{L_{w}^{p}} \leq C_{w}\|g(f)\|_{L_{w}^{p}}
$$


where $C_{w}=[w]_{A_{p / r}}^{1 / p}\left([w]_{A_{\infty}}^{\left(\frac{r}{2}-\frac{r}{p}\right)_{+}}+\left[w^{-\frac{r}{p-r}}\right]_{A_{\infty}}^{\frac{r}{p}}\right)^{\frac{1}{r}}$. In other words, $\left\|g_{d}(f)\right\|_{L_{w}^{p}} \leq$ $C_{w}\|f\|_{\mathbb{H}_{w}^{p}}$.

Proof Let us recall that

$$
g_{d}(f)(x)=\left(\sum_{j \in \mathbb{Z}} \sum_{Q \in \mathscr{D}_{j+N}}\left|\phi_{j} * f\left(x_{Q}\right)\right|^{2} \chi_{Q}(x)\right)^{\frac{1}{2}}
$$

where $x_{Q}$ is a fixed point in $Q$. According to the Calderón discrete reproducing formula (4.3)

$$
f(x)=\sum_{i \in \mathbb{Z}} \sum_{W \in \mathscr{D}_{i+N}}|W| \widetilde{\psi}_{i}\left(x-x_{W}\right) \psi_{i} * f\left(x_{W}\right)
$$

Then,

$$
\left(\phi_{j} * f\right)\left(x_{Q}\right)=\sum_{i \in \mathbb{Z}} \sum_{W \in \mathscr{D}_{i+N}}|W|\left(\phi_{j} * \widetilde{\psi}_{i}\right)\left(x_{Q}-x_{W}\right)\left(\psi_{i} * f\right)\left(x_{W}\right)
$$

Now, due to Lemma 2 we have

$$
\left|\left(\phi_{j} * \widetilde{\psi}_{i}\right)\left(x_{Q}-x_{W}\right)\right| \leq C 2^{-|j-i| a} \frac{2^{-(j \wedge i) b}}{\left(2^{-(j \wedge i)}+\left|x_{Q}-x_{W}\right|\right)^{n+b}}, \quad a, b \in \mathbb{N}
$$

Then, applying Lemma 3 with $a=b=M$, we get

$$
\begin{aligned}
\left|\left(\phi_{j} * f\right)\left(x_{Q}\right)\right| & \leq C \sum_{i \in \mathbb{Z}} \sum_{W \in \mathscr{D}_{i+N}}|W| \frac{2^{-|j-i| M} 2^{-(j \wedge i) M}}{\left(2^{-(j \wedge i)}+\left|x_{Q}-x_{W}\right|\right)^{n+M}}\left|\psi_{i} * f\left(x_{W}\right)\right| \\
& \leq C \sum_{i \in \mathbb{Z}} 2^{-|j-i| M_{2}} 2^{\left(\frac{1}{r}-1\right) n\left(N+(i-j)_{+}\right)} M_{r}\left(\sum_{W \in \mathscr{D}_{i+N}}\left|\psi_{i} * f\left(x_{W}\right)\right| \chi_{W}\right)(u) \\
& \leq C(N, M) \sum_{i \in \mathbb{Z}} 2^{-|j-i| M_{2}} 2^{\left(\frac{1}{r}-1\right) n(i-j)_{+}} M_{r}\left(\sum_{W \in \mathscr{D}_{i+N}}\left|\psi_{i} * f\left(x_{W}\right)\right| \chi_{W}\right)(u)
\end{aligned}
$$

where $u \in Q$. Then, by Hölder's inequality

$$
\left|\left(\phi_{j} * f\right)\left(x_{Q}\right)\right|^{2} \leq C \sum_{i \in \mathbb{Z}}\left(M_{r}\left(\sum_{W \in \mathscr{D}_{i+N}}\left|\psi_{i} * f\left(x_{W}\right)\right| \chi_{W}\right)(u)\right)^{2}\left(\sum_{i \in \mathbb{Z}} 2^{-2|j-i| M+\left(\frac{1}{r}-1\right) n(i-j)_{+}}\right) .
$$


Therefore, since $\sum_{Q \in \mathscr{D}_{j+N}} \chi_{Q}(x)=1$ for each $j \in \mathbb{Z}$, and the above inequality is independent of $Q$,

$$
\begin{aligned}
g_{d}(f)(x)= & \left(\sum_{j \in \mathbb{Z}} \sum_{Q \in \mathscr{D}_{j+N}}\left|\phi_{j} * f\left(x_{Q}\right)\right|^{2} \chi_{Q}(x)\right)^{\frac{1}{2}} \\
\leq & C\left[\sum_{i \in \mathbb{Z}}\left(M\left(\sum_{W \in \mathscr{D}_{i+N}}\left|\psi_{i} * f\left(x_{W}\right)\right| \chi_{W}\right)^{r}(u)\right)^{\frac{2}{r}}\right]^{\frac{r}{2} \cdot \frac{1}{r}} \\
& \times\left(\sum_{i, j \in \mathbb{Z}} 2^{-2|j-i| M+\left(\frac{1}{r}-1\right) n(i-j)_{+}}\right)^{\frac{1}{2}} \cdot
\end{aligned}
$$

It is easy to see that the double-series is convergent since $r>\frac{n}{n+M}$ and the sum only depends on $M$. Then, if we set $f_{i}=\left(\sum_{W \in \mathscr{D}_{i+N}}\left|\psi_{i} * f\left(x_{W}\right)\right| \chi_{W}\right)^{r}$, from Lemma 4 with $q=\frac{2}{r}>1$ in $L_{w}^{\frac{p}{r}}\left(\frac{p}{r}>1\right)$,

$$
\begin{aligned}
\left\|g_{d}(f)\right\|_{L_{w}^{p}} \leq C[w]_{A_{\frac{p}{r}}}^{\frac{r}{p}}\left([w]_{A_{\infty}}^{\left(\frac{r}{2}-\frac{r}{p}\right)_{+}}+[\sigma]_{A_{\infty}}^{\frac{r}{p}}\right)\left\|\left(\sum_{i \in \mathbb{Z}}\left(\sum_{W \in \mathscr{D}_{i+N}}\left|\psi_{i} * f\left(x_{W}\right)\right| \chi_{W}\right)^{2}\right)^{\frac{r}{2}}\right\|_{L_{w}^{\frac{p}{r}}} \\
=C[w]_{A_{\frac{p}{r}}^{\frac{r}{p}}}\left([w]_{A_{\infty}}^{\left(\frac{r}{2}-\frac{r}{p}\right)_{+}}+[\sigma]_{A_{\infty}}^{\frac{r}{p}}\right)\left\|\left(\sum_{i \in \mathbb{Z}}\left(\sum_{W \in \mathscr{D}_{i+N}}\left|\psi_{i} * f\left(x_{W}\right)\right| \chi_{W}\right)^{2}\right)^{\frac{1}{2}}\right\|_{L_{w}^{p}} .
\end{aligned}
$$

Notice that the above inequality holds for any $x_{W}$ in $W$, so we can take the infimum in each cube and then use that the dyadic cubes are disjoint in each level to get

$$
\begin{aligned}
\left\|g_{d}(f)\right\|_{L_{w}^{p}} & \leq C[w]_{A_{\frac{p}{r}}^{\frac{r}{p}}}^{\frac{r}{p}}\left([w]_{A_{\infty}}^{\left(\frac{r}{2}-\frac{r}{p}\right)_{+}}+[\sigma]_{A_{\infty}}^{\frac{r}{p}}\right)\left\|\left(\sum_{i \in \mathbb{Z}}\left(\sum_{W \in \mathscr{D} i_{i+N}} \inf _{x_{W} \in W}\left|\psi_{i} * f\left(x_{W}\right)\right| \chi_{W}\right)^{2}\right)^{\frac{1}{2}}\right\|_{L_{w}^{p}} \\
& \leq C[w]_{A_{\frac{p}{r}}^{\frac{r}{p}}}^{\frac{1}{p}}\left([w]_{A_{\infty}}^{\left(\frac{r}{2}-\frac{r}{p}\right)_{+}}+[\sigma]_{A_{\infty}}^{\frac{r}{p}}\right)\left\|\left(\sum_{i \in \mathbb{Z}}\left|\psi_{i} * f\right|^{2}\right)^{\frac{1}{2}}\right\|_{L_{w}^{p}} \\
& =C[w]_{A_{\frac{p}{r}}^{\frac{r}{p}}}^{\frac{r}{p}}\left([w]_{A_{\infty}}^{\left(\frac{r}{2}-\frac{r}{p}\right)_{+}}+[\sigma]_{A_{\infty}}^{\frac{r}{p}}\right)\|g(f)\|_{L_{w}^{p}} .
\end{aligned}
$$

The proof is now complete.

We are now in position to state the main result of this section, which gives us a precise quantitative bound for the operator norm on $\mathbb{H}_{w}^{p}$ (c.f. [22, Theorem 1.1]). 
Theorem 7 Let $T$ be a singular integral of convolution type with kernel $K$ satisfying conditions (4.1) and (4.2). Let $w \in A_{\infty}$ and $0<p<\infty$. Then,

$$
\|T f\|_{\mathbb{H}_{w}^{p}} \leq C[w]_{A_{\frac{p}{r}}}^{\frac{r}{p}}\left([w]_{A_{\infty}}^{\left(\frac{r}{2}-\frac{r}{p}\right)_{+}}+[\sigma]_{A_{\infty}}^{\frac{r}{p}}\right)\|f\|_{\mathbb{H}_{w}^{p}}
$$

where r satisfies $\frac{n}{n+M}<r<\min \left\{\frac{p}{q_{w}}, 1\right\}$.

Proof Since $L^{2} \cap \mathbb{H}_{w}^{p}$ is dense in $\mathbb{H}_{w}^{p}$, by standard density arguments, we assume $f \in L^{2} \cap \mathbb{H}_{w}^{p}$. The proof is very similar to the proof of Lemma 5. By Calderón's reproducing formula (4.3)

$$
\begin{aligned}
g(T f)(x) & =\left(\sum_{j \in \mathbb{Z}} \sum_{Q \in \mathscr{D}_{j+N}}\left|\psi_{j} * K * f(x)\right|^{2} \chi_{Q}(x)\right)^{1 / 2} \\
& =\left[\sum_{j, Q}\left|\psi_{j} * K *\left(\sum_{i \in \mathbb{Z}} \sum_{W \in \mathscr{D}_{i+N}}|W| \tilde{\psi}_{i}\left(x-x_{W}\right) \psi_{i} * f\left(x_{W}\right)\right)\right|^{2} \chi_{Q}(x)\right]^{1 / 2} \\
& =\left[\sum_{j, Q}\left|\left(\sum_{i, W} \psi_{j} * K * \widetilde{\psi}_{i}\left(x-x_{W}\right)|W| \psi_{i} * f\left(x_{W}\right)\right)\right|^{2} \chi_{Q}(x)\right]^{1 / 2} .
\end{aligned}
$$

From the properties on $K, K * \widetilde{\psi}_{i} \in \mathscr{S}_{M}$, so we can apply the almost orthogonality property (Lemma 2) to $K * \widetilde{\psi}_{i}$ and $\psi_{j}$ with $a=b=M$. Then, as in the proof of Lemma 5, we use Lemma 3, Hölder's inequality, the properties of the dyadic cubes and the restriction on $r$ to get

$$
g(T f)(x) \leq C\left(\sum_{i \in \mathbb{Z}}\left(M\left(\sum_{W \in \mathscr{D}_{i+N}}\left|\psi_{i} * f\left(x_{W}\right)\right| \chi_{W}(x)\right)^{r}\right)^{\frac{2}{r}}\right)^{\frac{1}{2}}
$$

and now the proof continues in the same way. Thus, from Lemma 4 we obtain the following quantitative bound

$$
\|T f\|_{\mathbb{H}_{w}^{p}} \leq C[w]_{A_{\frac{p}{r}}}^{\frac{r}{p}}\left([w]_{A_{\infty}}^{\left(\frac{r}{2}-\frac{r}{p}\right)_{+}}+[\sigma]_{A_{\infty}}^{\frac{r}{p}}\right)\|g(f)\|_{L_{w}^{p}}=\|f\|_{\mathbb{H}_{w}^{p}} .
$$

Acknowledgements María Eugenia wants to mention that the study of these estimates was pointed out by Sheldy Ombrosi, her supervisor during postdoctoral research, which was done under a fellowship granted by CONICET, Argentina. Also, she would like to thank Carlos Pérez, Cristina Pereyra and Michael Wilson for their help and interest when contacting them. 
Funding First author was supported by Universidad de Buenos Aires (Grant No. 20020120100050), by Agencia Nacional de Promoción Científica y Tecnológica (Grant No. PICT 2014-1771) and by Universidad Nacional de La Plata (Grant No. UNLP 11/X752 and UNLP 11/X805). Second author was supported by Universidad Nacional del Litoral (Grants No. CAI+D 2015-026 and CAI+D 2015-066).

\section{References}

1. Buckley, S.M.: Estimates for operator norms on weighted spaces and reverse Jensen inequalities. Trans. Am. Math. Soc. 340(1), 253-272 (1993)

2. Cejas, M.E., Li, K., Pérez, C., Rivera-Ríos,I.P.: A sparse approach to sharp weighted estimates for vector-valued operators. Sci. China Math. (in press). Preprint available at arXiv:1712.05781

3. Cruz-Uribe, D., Martell, J.M., Pérez, C.: Extrapolation from $A_{\infty}$ weights and applications. J. Funct. Anal. 213(2), 412-439 (2004)

4. Cruz-Uribe, D., Martell, J.M., Pérez, C.: Sharp weighted estimates for classical operators. Adv. Math. 229(1), 408-441 (2012)

5. Dragičević, O., Grafakos, L., Pereyra, M.C., Petermichl, S.: Extrapolation and sharp norm estimates for classical operators on weighted Lebesgue spaces. Publ. Mat. 49(1), 73-91 (2005)

6. Ding, Y., Yongsheng, H., Lu, G., Wu, X.: Boundedness of singular integrals on multiparameter weighted Hardy spaces $H_{w}^{p}\left(\mathbb{R}^{n} \times \mathbb{R}^{m}\right)$. Potential Anal. 37(1), 31-56 (2012)

7. Dindoš, M., Wall, T.: The sharp $A_{p}$ constant for weights in a reverse-Hölder class. Rev. Mat. Iberoam. 25(2), 559-594 (2009)

8. Frazier, M., Jawerth, B.: Decomposition of Besov spaces. Indiana Univ. Math. J. 34(4), 777-799 (1985)

9. García-Cuerva, J.: Weighted Hardy spaces. In: Harmonic Analysis in Euclidean Spaces (Proc. Sympos. Pure Math., Williams Coll., Williamstown, Mass., 1978), Part 1

10. Gundy, R.F., Wheeden, R.L.: Weighted integral inequalities for the nontangential maximal function, Lusin area integral, and Walsh-Paley series. Stud. Math. 49, 107-124 (1973/74)

11. Hytönen, T.P., Lacey, M.T., Pérez, C.: Sharp weighted bounds for the $q$-variation of singular integrals. Bull. Lond. Math. Soc. 45(3), 529-540 (2013)

12. Hart, J., Oliveira, L.: Hardy space estimates for limited ranges of Muckenhoupt weights. Adv. Math. 313, 803-838 (2017)

13. Hytönen, T., Pérez, C.: Sharp weighted bounds involving $A_{\infty}$. Anal. PDE 6(4), 777-818 (2013)

14. Hagelstein, P., Parissis, I.: Weighted Solyanik estimates for the Hardy-Littlewood maximal operator and embedding of $\mathscr{A}_{\infty}$ into $\mathscr{A}_{p}$. J. Geom. Anal. 26(2), 924-946 (2016)

15. Hytönen, T.P.: The sharp weighted bound for general Calderón-Zygmund operators. Ann. Math. (2), 175(3):1473-1506 (2012)

16. Korey, M.B.: Ideal weights: asymptotically optimal versions of doubling, absolute continuity, and bounded mean oscillation. J. Fourier Anal. Appl. 4(4-5), 491-519 (1998)

17. Kurtz, D.S., Wheeden, R.L.: Results on weighted norm inequalities for multipliers. Trans. Am. Math. Soc. 255, 343-362 (1979)

18. Lee, M.-Y.: Calderón-Zygmund operators on weighted Hardy spaces. Potential Anal. 38(3), 699-709 (2013)

19. Lerner, A.K.: On some weighted norm inequalities for Littlewood-Paley operators. Illinois J. Math. 52(2), 653-666 (2008)

20. Lee, M.-Y., Lin, C.-C.: The molecular characterization of weighted Hardy spaces. J. Funct. Anal. 188(2), 442-460 (2002)

21. Lerner, A.K., Ombrosi, S., Pérez, C.: Sharp $A_{1}$ bounds for Calderón-Zygmund operators and the relationship with a problem of Muckenhoupt and Wheeden. Int. Math. Res. Not. IMRN, (6):Art. ID rnm161, 11 (2008)

22. Lu, G., Zhu, Y.: Bounds of singular integrals on weighted Hardy spaces and discrete Littlewood-Paley analysis. J. Geom. Anal. 22(3), 666-684 (2012)

23. Mitsis, T.: Embedding $B_{\infty}$ into Muckenhoupt classes. Proc. Am. Math. Soc. 133(4), 1057-1061 (2005)

24. Meda, S., Sjögren, P., Vallarino, M.: Atomic decompositions and operators on Hardy spaces. Rev. Un. Mat. Argentina 50(2), 15-22 (2009)

25. Petermichl, S.: The sharp bound for the Hilbert transform on weighted Lebesgue spaces in terms of the classical $A_{p}$ characteristic. Am. J. Math. 129(5), 1355-1375 (2007) 
26. Petermichl, S.: The sharp weighted bound for the Riesz transforms. Proc. Am. Math. Soc. 136(4), 1237-1249 (2008)

27. Politis, A.: Sharp results on the relation between weight spaces and BMO. ProQuest LLC, Ann Arbor, MI, 1995. Thesis (Ph.D.), The University of Chicago

28. Petermichl, S., Pott, S.: An estimate for weighted Hilbert transform via square functions. Trans. Am. Math. Soc. 354(4), 1699-1703 (2002)

29. Petermichl, S., Volberg, A.: Heating of the Ahlfors-Beurling operator: weakly quasiregular maps on the plane are quasiregular. Duke Math. J. 112(2), 281-305 (2002)

30. Strömberg, J.-O., Torchinsky, A.: Weighted Hardy Spaces. Lecture Notes in Mathematics, vol. 1381. Springer, Berlin (1989)

31. Stein, E.M.: Singular integrals and differentiability properties of functions. Princeton Mathematical Series, No. 30. Princeton University Press, Princeton, N.J. (1970)

32. Wik, I.: On Muckenhoupt's classes of weight functions. Stud. Math. 94(3), 245-255 (1989)

33. Wilson, J.M.: Weighted inequalities for the square function. In: Commutative harmonic analysis (Canton, NY, 1987), volume 91 of Contemp. Math., pp. 299-305. Amer. Math. Soc., Providence (1989)

34. Wilson, J.M.: Chanillo-Wheeden inequalities for $0<p \leq 1$. J. Lond. Math. Soc. (2), 41(2):283-294 (1990)

35. Wilson, M.: Weighted Littlewood-Paley Theory and Exponential-Square Integrability. Lecture Notes in Mathematics, vol. 1924. Springer, Berlin (2008)

36. Wang, H., Liu, H.: The intrinsic square function characterizations of weighted Hardy spaces. Illinois J. Math. 56(2), 367-381 (2012)

Publisher's Note Springer Nature remains neutral with regard to jurisdictional claims in published maps and institutional affiliations. 